\title{
FORAREX - Designing a Life-Support System for Microbiological Research aboard a Sounding Rocket
}

\author{
Nils Kunst ${ }^{1,3}$, Greta Sondej ${ }^{1}$, \\ Kay Menken-Siemers ${ }^{1}$, Niklas Kipry ${ }^{1}$, Daniel Ripberger ${ }^{1}$, Jan Blumenkamp ${ }^{1}$, Steffen Lohmann ${ }^{1}$, \\ Christoph Kulmann ${ }^{1}$, Scarlett Gac Cáceres ${ }^{1}$, Diren Senger ${ }^{1}$, Claudia Bruhn ${ }^{1}$, \\ Katrin Hättig ${ }^{1}$, Sebastian Gfellner ${ }^{1}$ and \\ Johanna Hartmann ${ }^{1,2}$ \\ ${ }^{1}$ University Bremen, 28359 Bremen, Germany \\ ${ }^{2}$ University of the Arts Bremen, 28217 Bremen, Germany
}

\begin{abstract}
Goal of the FORAREX (FORAminifera Rocket EXperiment) project is the proof of concept for a rocket and space suitable life support system for foraminifera Amphistegina lobifera with integrated scientific sensors. Amphistegina lobifera is a unicellular marine protist with an external calcareous shell and diatoms as endosymbiotic algae. This first experiment demonstrates that the technical setup is feasible and works fully automated.
\end{abstract}

This paper has been submitted in a similar version elsewhere.

Keywords - foraminifera, microgravity, cell physiology and motility, life-support-system, sounding rocket, REXUS/BEXUS programme, education

\section{INTRODUCTION}

FORAREX (FORAminifera Rocket EXperiment) was developed by an interdisciplinary team of students from the University of Bremen. The technical goal of the FORAREX project is to proof the concept for a rocket and space suitable life support systems for the foraminifera Amphistegina lobifera with integrated scientific sensors. The used species Amphistegina lobifera is a unicellular marine protist with an external calcareous shell. In addition, it hosts diatoms as endosymbionts which photosynthesise. The experiment started three days before launch and continued for four weeks. During this period the sensors took pictures of the foraminifera and measured the water parameters. Moreover the water in the experiment setup was labelled to in order to distinguish the shell sections that have been grown before, during and after the rocket flight phase. The shell will be analysis by means of scanning electron microscopy. The biological goal of the experiment is to investigate the effect of microgravity on the motility and shell biomineralisation process of the foraminifera.

\section{MATERIAL AND METHODS}

\section{A. Overview}

The bases of the experiment design were the biological and the predefined REXUS/BEXUS manual requirements. First the basic needs of the foraminifera have to be covered: An observation chamber (FlowCell) with sea water to life in. The Temperature of the water should be around $20^{\circ} \mathrm{C}$ but shall be in the range of $15{ }^{\circ} \mathrm{C}$ to $30{ }^{\circ} \mathrm{C}$. The pressure in the FlowCell should be around 1 bar to avoid outgassing of the water. The water in the FlowCell shall be circulated to avoid high concentration of metabolic products. The artificial light shall support the photosynthesis of the endosymbionts. The diurnal cycle shall be 12 hours day and 12 hours night. To ensure these needs the foraminifera had to keep under laboratory conditions as long as possible. Because of this the containment with the foraminifera, water circle and integrated sensors has to be inserted via Late Access. As a next step, the scientific goals have to be fulfilled: To observe the motility and shape of the foraminifera a camera has to be integrated. To monitor the respiration and photosynthesis an oxygen sensor has to be included as well. Further the $\mathrm{pH}$ level has to be monitored. Because of the long experiment time, optical sensors are used. These sensors need temperature and pressure measurements to compensate their values. Moreover a biological filter was added in front of the area of the sensor spots to avoid a biological film, which could alter the measurements. To allow water manipulation a direct, leakage free access to the main water storage and the FlowCell has to be implemented. To increase the scientific output, the setup is doubled. Finally the REXUS/BEXUS requirements [1]: The experiment always has to be able to answer the service module. The experiment has to contain water absorbent material for twice the amount of water used. The experiment consists of two setups: The Late Access Module and the REXUS Module. The pressurised Late Access Module (LA Module) contains the two water circuits with the foraminifera, life support system (temperature, water circulation and light) and scientific sensors (camera, oxygen and $\mathrm{pH}$ ) together with water absorption material.

\section{B. Mechanic}

The core of the LA Module is the stereolithographic printed FlowCell for the foraminifera. The observation chamber embedded inside holds the foraminifera in the camera field of view. To retain the picture sharpness the height of the inner observation chamber is minimized to $2 \mathrm{~mm}$. This height depends on the insertion mechanism. To allow water flow but hold the foraminifera inside the observation chamber is partitioned by bars. The cameras shoot through a polycarbonate disc into the chamber. After watering and deflating the setup, the foraminifera are injected. This is done with a cannula (15 gauge; point style 5) through a triple septa and a moveable blocker into the observation chamber. The rotatable blocker prevents the foraminifera from moving into the injection canal, which is outside the area of camera observation. Rolling membranes keep the water pressure constant during injections or extractions. The water circuits also include the water 
reservoir with integrated oxygen and $\mathrm{pH}$ optodes including a thermal sensor and a deflation support. Peristaltic water pumps generate flow.

\section{Thermal}

Due to the biological part of the experiment, the temperature does not only have to fit during flight for measurements. The temperature has to fit at all times to ensure the survival of the organisms. The challenge of this design is to allow enough heat outflow to prevent overheating while the integrated experiment is in a warm environment (e.g. the laboratory or the heated launcher). At the same time, it has to keep enough heat for the time it lay in the snow after landing. To compensate the necessary thermal outflow, an active heater is added to the insulation.

\section{Electronic}

The controller of the experiment is a Raspberry Pi Compute Module 3. The two cameras are Raspberry Pi Cameras v2 (8 MP). The oxygen sensors in the two water cycles are Pyroscience Piccolo OEM. The combined $\mathrm{pH}$ and oxygen sensor in one of the water cycle is a prototype supplied by PreSens. For temperature measurements in the water, a PT100 is used. For air pressure measurements a MS580305BA01-00 is used.

\section{E. Software}

The Raspberry Pi Compute Module 3 run by Raspbian a Linux OS especially for Raspberry Pi Boards. It handles the data management and includes the basic drivers. The control program for the sensors and actuators is written in $\mathrm{C}++$. It communicates via the REXUS Service Module with a ground control station for monitoring. The ground control station is written with QT.

\section{$F$. Chemicals and foraminiferas}

The foraminifera species Amphistegina lobifera was provided by the AG Kucera of the Marum, Bremen. The used ones are sized around $1.3 \mathrm{~mm}$. The species hosts diatoms as endosymbionts. The standard artificial seawater for cultivation was Tropic Marin ${ }^{\circledR}$ CLASSIC SEA SALT which was modified by injected labels like strontium chloridehexahydrate. The injections were always done in a laboratory.

\section{G. Sounding rocket and flight}

The REXUS 25 rocket was equipped with an "Improved Orion" motor. On board were five experiments (FLOMESS, HEDGEHOG, PR ${ }^{3}$, FORAREX and GAME). The weight of the setup was $541 \mathrm{~kg}$ by $5927 \mathrm{~mm}$ length. The lift-off was on March 11 $1^{\text {th }}, 2019$ at 09:20 UTC. The altitude was at least $82.184 \mathrm{~km}$ with around two minutes of microgravity. The late access module was inserted after start of countdown at T-170 min. The functionality of the sensors was checked by uplink/downlink communication before lift-off.

\section{RESULTS}

The injection of the foraminifera and the labels were successful. The Late Access was done on schedule.

The cameras worked nominal during flight as well as the other sensors. The only exception was the PyroScience oxygen sensor of water circle 0 . After recovery we found the fiberglass cable was broken. The electronics worked nominal. The ground control station also worked nominal. During flight the sensors recorded a pressure decrease from 1000 mbar to around 530 mbar. During flight the temperature increase, the concentration of oxygen within the water decreased and the $\mathrm{pH}$ value increased slightly. After recovery the temperature was at $14.319{ }^{\circ} \mathrm{C}$. During the week after launch a leakage of water was detected. All known weak points were additionally sealed with silicon. But the leakage continued. After disassembling it turned out the leakage was caused by broken pump tubing. This was in contrast to the conducted tests. Moreover while disassembling the foraminifera of FlowCell 0 did not stay in the dedicated area. This was in contrast to the conducted tests, too. It was found in the tube connected to the FlowCell. The inner parts of the setup were very clean without noticeable biofilm growth at disassembly. A ground control experiment was done after the flight campaign. In this the pump tubing broke again and one foraminifera slipped into the tube as happened during the flight experiment. Everything else worked nominally. Further vitality, shell and water analysis of both experiments will be published in a separate paper.

\section{DISCUSSION AND CONCLUSION}

The broken fiberglass cable of water cycle 0 caused disturbances in the oxygen measurements. Luckily there was a second oxygen sensor (Presens) in this water cyrcle. So after repair it considered as a systematic failure. The pressure decrease during flight was still above the water boiling pressure point. The temperatures during and after launch were in accordance with survival of the organisms.

All in all this first experiment demonstrates that such a technical setup is possible and feasible. Off course the setup need further improvements for follow-on missions. For the all occurred problems solution possibilities with high potential are already in mind.

\section{ACKNOWLEDGMENT}

We acknowledge the REXUS/BEXUS programme namely the German Aerospace Center (DLR), the Swedish National Space Agency (SNSA) and the European Space Agency (ESA). A special thank goes to the ZARM (Center of Applied Space Technology and Microgravity) for their outstanding support.

We also want to thank our consulting and material supporters from aerospace namely Airbus, the German Research Center for Artificial Intelligence Robotics Innovation Center (DFKI RIC Bremen) and the OHB Systems AG. The same applies to our Marine Research supporters Alfred Wegener Institute, MARUM (Center for Marine Environmental Science) and the Max Planck Institute for Marine Microbiology. Moreover we want to thank our University especially the NW1 training workshop for their support.

Finally we want to thank our industrial partners BuyIsotope Neonest AB, OMNILAB-LABORZENTRUM GmbH \& Co.KG, PreSens Precision Sensing GmbH, PyroScience $\mathrm{GmbH}$, Watson-Marlow GmbH Fluid Technology Group and ZF Friedrichshafen AG (training centre Lemförde).

\section{REFERENCES}

[1] EuroLaunch: REXUS User Manual Version 7.15 November 2017 